\title{
An Improved Graph Method for Linear Goal Programming
}

\author{
Xisheng Yu \& Chuanyi Luo \\ School of Mathematics \\ Southwestern University of Finance and Economics \\ Chengdu 610074, China \\ E-mail: yuxisheng@swufe.edu.cn
}

This work was supported by the Scientific Research Fund of Southwestern University of Finance and Economics (No. QN0819)

\begin{abstract}
The traditional Graph Method is a basic approach to solve Linear Goal Programming. The paper attained a modified method based on the traditional way. The modified method can attain the satisfaction solution, while the traditional Graph Method has its limitation in solving some Linear Goal Programming.
\end{abstract}

Keywords: Linear Goal Programming, Graph Method

\section{Introduction}

Goal programming is one of the most widely used methodologies in operations research and management science, and it encompasses most classes of multiple objective programming models. Goal programming is first brought up in 1961 by A. Charnes and W. W. Cooper (Editor Group of O.R., 1990), and then there were many scholars studying it and it developed rapidly in the past several decades. Goal programming allowed a decidemaker to put many requests within one decision. With goal programming the decide-maker should not look for an absolute optimal solution, instead, he should only look for a solution that can make himself more satisfactory than any other solutions. Because goal programming makes up some defects of linear programming, it is considered as a decision tool that is nearer to real decision process than linear programming (Hu Yunquan, 2003).

In 1972, Lee.Sang.M put forward the graph method for linear goal programming for the first time in his monograph 'Goal Programming for Decision Analysis (Editor Group of O.R., 1990). In the last 30 years, the graph method was included as an important content of linear goal programming in many textbooks, such as in (Hu Yunquan, 2003, Ignizio, 1976. Lee and Sang M., 1972). Analogous to the graph method for liner programming, the graph method for goal programming can only solve problems with no more than two decision variables. Since there are lots of problems with no more than two decision variables and graph method can give us some help on understanding the characteristics of the optimal solution of linear programming and the satisfaction solution of linear goal programming, graph method was an important part in almost all textbooks that included goal programming. Hence it's of theoretic sense to study graph methods for goal programming.

\section{Goal programming}

We describe linear goal programming in this section. Goal programming is a programming problem with multiple goals, in which there is a priority order among the goals. We first introduce some basic notations used commonly in goal programming as follows:

\subsection{Deviation variable}

For each decision goal, we introduce a positive deviation variable $d^{+}$and a negative deviation variable $d^{-}$, while $d^{+}$denotes how much the decision has exceeded the goal, and $d^{-}$denotes how far the decision is from the goal. 
Here, we have $d^{+} \geq 0, d^{-} \geq 0$, and $d^{+} \bullet d^{-}=0$.

\subsection{Absolute constraint and goal constraint}

If a constraint must be satisfied, we call it absolute constraint. Because it is a hard constraint, a solution is not a feasible one if it can not satisfy any one absolute constraint. Goal constraint is a special weak constraint that can only be seen in goal programming. And sometimes a feasible solution does not satisfy a goal constraint.

\subsection{Priority factor and weight coefficient}

Some goal is important, while some others are unimportant. If a goal is far more important than another one, we give it a priority factor $P_{l}$, and we give another goal a priority factor $P_{l+1}$. Here we have $P_{l}>>P_{l+1}$. If a goal is a little more important than another one, we can give it a bigger weight coefficient while we give them same priority factor.

\subsection{Goal function}

Goal function is composed of goal constraints' deviation variables, their priority factors and weight coefficient. Usually, goal programming try to minimize its deviation variables, such as $\min \left\{f\left(d^{+}+d^{-}\right)\right\}, \min \left\{f\left(d^{+}\right)\right\}$and $\min \left\{f\left(d^{-}\right)\right\}$.

\subsection{Satisfaction solution}

If a solution satisfies all the absolute constraints, and its cost value is no bigger than any other solution, we call it a satisfaction solution.

\section{Traditional graph method for linear goal programming}

While constraints and cost function are all linear, goal programming is linear goal programming.

In traditional graph method for linear goal programming, firstly the feasible solutions should satisfy all absolute constraints. Then we consider every goal constraint according to their priority factors. Generally, if $R_{j}$ is the solution region for priority factor $P_{j}$, solution region $R_{j+1}$ for priority factor $P_{j+1}$ must be a subspace of $R_{j}$, i.e. $R_{j+1} \subseteq R_{j}$. If $R_{j} \neq \Phi$, and $R_{j+1}=\Phi$, there is a satisfaction solution in $R_{j}$. It satisfies goal $P_{1}, P_{2}, \cdots, P_{j}$, but it can not satisfy the other goals always.

Since there is no solution that can satisfy all the goals of $P_{j+1}$, we preferentially let deviation variable be zero whose cost coefficient is larger. For example, if the goal function is $2 d_{3}^{-}+3 d_{4}^{-}$, we let $d_{4}^{-}$be zero preferentially as the coefficient 3 of $d_{4}^{-}$is larger than that 2 of $d_{3}^{-}$. So the satisfaction solution satisfies $d_{4}^{-}=0$.

The above described method is just the Graph Method for Linear Goal Programming. But when we look for a satisfaction solution simply by whether the coefficient is larger or smaller, we ignore the difference among the influence that the constraint functions have upon different deviation variables, and so it is not reliable. Now let us look at an example of goal programming and solve it by Graph Method:

Example1: $\min \left\{P_{1} d_{1}^{-}, P_{2} d_{2}^{+}, P_{3}\left(2 d_{3}^{-}+3 d_{4}^{-}\right), P_{4} d_{1}^{+}\right\}$

$$
\text { st. }\left\{\begin{array}{lc}
x_{1}+2 x_{2}+d_{1}^{-}-d_{1}^{+} & =6 \\
x_{1}+2 x_{2} \quad+d_{2}^{-}-d_{2}^{+} & =9 \\
x_{1}-2 x_{2} & +d_{3}^{-}-d_{3}^{+} \\
x_{1}-2 x_{2} & =4 \\
x_{1}, x_{2}, d_{i}^{-}, d_{i}^{+} \geq 0, d_{i}^{-} * d_{i}^{+}=0, \quad i=1,2,3,4 & =2
\end{array}\right.
$$

From Fig. 1, we know that the solution region $R_{2}$ for goal $P_{1}$ and $P_{2}$ is quadrangle ABCD. While considering the goal $P_{3}$, we minimize $d_{4}^{-}$in priority as its cost coefficient 3 is larger than that 2 of $P_{3}$. So the feasible solution region is reduced to quadrangle CDEF. Then we minimize $d_{3}^{-}$. But there is no point that satisfies $d_{3}^{-}=0$ in quadrangle CDEF, so we have to try to look for a point that minimize $d_{3}^{-}$. The point is $\mathbf{F}(\mathbf{5 , 2})$. So the feasible solution for the Goal Programming is $x_{1}=5$, and $x_{2}=2$.

\section{Defect of tradition graph method}

In fact, the cost value of point $\mathbf{F}(\mathbf{5}, \mathbf{2})$ about $P_{3}\left(2 d_{3}^{-}+3 d_{4}^{-}\right)$is 3 , and that of $\mathbf{G}(\mathbf{6 . 5}, \mathbf{1 . 2 5})$ is 2.25 which is smaller than 3. So point $\mathbf{G}(\mathbf{6 . 5}, \mathbf{1 . 2 5})$ is more satisfactory than point $\mathbf{F}(\mathbf{5}, \mathbf{2})$, and the satisfaction solution is $x_{1}=6.5$, and $x_{2}=1.25$ indeed. 
With the above analysis, we can get that the solution found by the traditional graph method is not very satisfactory sometimes. Now let us prove that point $\mathbf{G}(\mathbf{6 . 5}, \mathbf{1 . 2 5})$ is the satisfaction solution of the example.

Theorem1: $\mathbf{G}(\mathbf{6 . 5}, \mathbf{1 . 2 5})$ is the satisfaction solution of example1.

Proof: First, we will prove that any point I out of quadrangle EFGH must be not a satisfaction solution. Without loss of generality, we suppose that point $\mathbf{I}$ is in quadrangle ABGH. Firstly, we move point $\mathbf{I}$ to line $d_{3}^{+}=d_{3}^{-}=0$ in the vertical direction of line $d_{4}^{+}=d_{4}^{-}=0$, and the point of intersection is denoted by $\mathbf{I}$. Obviously, $d_{3}^{-}\left(I^{\prime}\right)=0<d_{3}^{-}(I)$, and $d_{4}^{-}\left(I^{\prime}\right)<d_{4}^{-}(I)$ so we have $\left(2 d_{3}^{-}+3 d_{4}^{-}\right)\left(I^{\prime}\right)<\left(2 d_{3}^{-}+3 d_{4}^{-}\right)(I)$.

But $d_{3}^{-}(G)+3 d_{3}^{-}\left(I^{\prime}\right)=0$, and $d_{4}^{-}(G)<d_{4}^{-}\left(G^{\prime}\right)$, so

$$
\begin{aligned}
\left(2 d_{3}^{-}+3 d_{4}^{-}\right)(G) & <\left(2 d_{3}^{-}+3 d_{4}^{-}\right)\left(I^{\prime}\right) \\
& <\left(2 d_{3}^{-}+3 d_{4}^{-}\right)(I)
\end{aligned}
$$

That is to say, point $\mathbf{G}$ is more satisfactory than point $\mathbf{I}$. With same reason, point $\mathbf{F}$ is more satisfactory than all the points in quadrangle CDEF.

Now let us prove that the cost value of point $\mathbf{G}$ is smaller than that of all points in quadrangle EFGH. In quadrangle EFGH, all the points satisfy that $d_{1}^{-}=0, d_{2}^{+}=0, d_{3}^{+}=0$ and $d_{4}^{+}=0$, so the original problem can be transferred into the following linear programming:

$$
\text { s.t. } \begin{cases}\min \left\{2 d_{3}^{-}+3 d_{4}^{-}\right\} & =6 \\ x_{1}+2 x_{2}-d_{1}^{+} & =9 \\ x_{1}+2 x_{2}+d_{2}^{-} & =4 \\ x_{1}-2 x_{2}+d_{3}^{-} & =2 \\ x_{1}-2 x_{2}+d_{4}^{-} & \\ x_{1}, x_{2}, d_{i}^{-}, d_{i}^{+} \geq 0, \quad i=1,2,3,4 & \end{cases}
$$

The optimal solution of the above linear programming certainly can be found in the vertices of its feasible region, and they are $\mathbf{E}, \mathbf{F}, \mathbf{G}$ and $\mathbf{H}$. In the four vertices, the cost value of point $\mathbf{F}$ is obviously smaller than that of point $\mathrm{E}$, and that of point $\mathrm{G}$ is smaller than that of point $\mathrm{H}$. And with the above calculation, we know that the cost value of point $\mathbf{G}$ is smaller than that of point $\mathbf{F}$. So point $\mathbf{G}$ is the most satisfactory point, and point $\mathbf{G}(\mathbf{6 . 5}$, 1.25) is the satisfaction solution of Example1.

\section{Improvement of graph method}

With the proof of Theorem 1, we know that there must be a vertex which is the satisfaction solution of the goal programming. So we can improve the Traditional Graph Method. We put a concept algorithm of the improved graph method as follows:

Suppose that the feasible set of goal $P_{1}, P_{2}, \cdots, P_{j}$ is $R_{j}$, and there is no point that can satisfy all the goals in $R_{j}$. Then, for every deviation variable in $P_{j+1}$, we first draw a line whose deviation variables are all equal to zero rightly, then we calculate the intersection of each line and the boundary of $R_{j}$ and the intersection of any two lines among them. Successively we calculate all the points' cost function of $P_{j+1}$. The point whose cost function is the smallest is just the satisfaction solution.

Remark 1: In Example 1, there is no point that can make all deviation variables in goal $P_{3}\left(2 d_{3}^{-}+3 d_{4}^{-}\right)\left(d_{3}^{-}\right.$and $d_{4}^{-}$) equal to zero in $R_{2}$ (quadrangle $\mathbf{A B C D}$ ). Then we draw the lines $d_{3}^{+}=d_{3}^{-}=0$ and $d_{4}^{+}=d_{4}^{-}=0$. Following the steps described in the above concept algorithm, we find the satisfaction solution $\mathrm{G}$ as prove in the previous section.

Remark 2: The concept algorithm posed above can find a satisfaction solution, because the satisfaction must be on the intersections among the lines of the deviation variables which are all zeros and the boundary of the feasible region of the problem.

\section{Conclusion}

The Improved Graph Method for Goal Programming can solve problems whose goals are to minimize their deviation variables with no more than two decision variables. While it can not solve the problems whose goals are to maximize their deviation variables, such as $\max 2 d_{2}^{+}+3 d_{3}^{-}$and $\min 2 d_{2}^{+}-3 d_{3}^{-}$. 


\section{References}

Editor Group of O.R. (1990). Operation Research (Modified Edition). Beijing: Tsinghua University Press.

$\mathrm{Hu}$ Yunquan and Guo Yaohuang (2003). Operation Research (the 2nd edition). Beijing: Tsinghua University Press.

Ignizio and James P. (1976). Goal Programming and Extensions. D.C.Heath and Company.

Lee and Sang M. (1972). Goal Programming for Decision Analysis. Philadelphia: Auerbach Publishers.

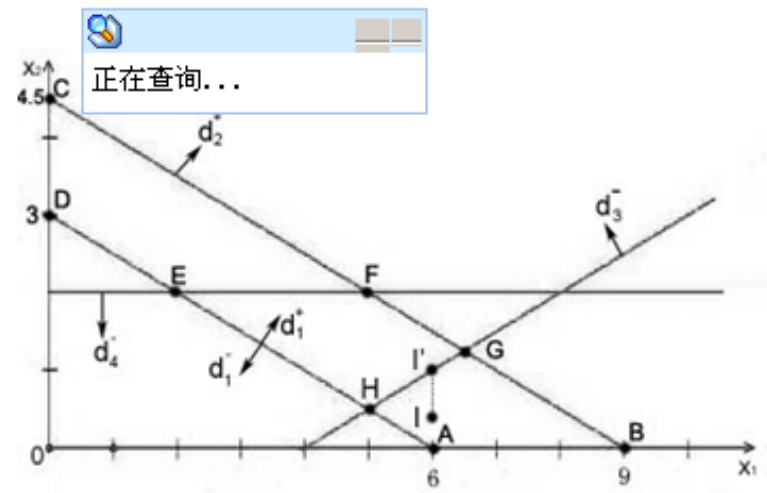

Figure 1. solution of Example 1 